\title{
The Relationship of 2D:4D Ratio and Hand Grip Strength to the Incidence of Multiple Sclerosis
}

\author{
Hamid Arazi' ${ }^{1}$, Roghayeh Bavafa Birak Olia' ${ }^{1}$, Shahriar Nafissi ${ }^{2}$, Nahid Beladi Moghadam ${ }^{3}$, Akram Falahati ${ }^{4}$ \\ 'Department of Exercise Physiology, Faculty of Sport Sciences, University of Guilan, Rasht, Iran, ${ }^{2}$ Department of Neurology, School of Medicine, \\ Tehran University of Medical Sciences, Tehran, Iran, ${ }^{3}$ Department of Neurology, School of Medicine, Shahid Beheshti University of Medical Sciences, \\ Tehran, Iran, ${ }^{4}$ Department of Exercise Physiology, University Campus, University of Guilan, Rasht, Iran
}

\begin{abstract}
Regarding the muscular weakness in patients with Multiple and the importance of the 2nd digit to 4th digit (2D:4D) ratio as a representative of the androgen level in women, the aim of this study was to investigate the relationship between level of physical activity, handgrip strength and 2D:4D ratio with the incidence of multiple sclerosis. This cross-sectional study was conducted on 100 female patients with average age of 35.01 years, BMl $24.04 \mathrm{~kg} / \mathrm{m}^{2}$ and disability development scale of Kurtzke (EDSS) equal to 8.5-0 among the patients of Shariati, Imam Hossein and Rasul Akram hospitals in Tehran in time intervals of December 2015 to June 2016. Hand grip strength variables were measured using the dynamometer, the 2D:4D ratio was measured with a digital calliper and the participants' physical activity were measured using the Beck physical activity questionnaire and EDSS of patients was determined by a Neurologist. The study data was analysed using statistical methods of Pearson and Spearman correlation analysis. There was a significant relationship between the physical activity level and EDSS $(P<0.01)$ and the handgrip strength with the EDSS level $(P<0.05)$ in MS patients. However, there was no significant relationship between the 2D:4D ratio and EDSS level. Based on these results, it seems that, the increase of physical activity level and the hand grip strength is associated with a reduced level of disability in patients with multiple sclerosis. Therefore, it is recommended to prescribe an exercise program which is in accordance with MS patients' condition.
\end{abstract}

Key words: Physical activity, Multiple sclerosis, 2D:4D Ratio, Hand grip strength, EDSS

\section{Introduction}

Today, the increasing prevalence of chronic diseases in the world has added to the importance of issues such as quality of life, health care costs and lack of staff available to respond to the existing demands on health services) Mohr \& Pelletier, 2006; Moy, Chang \& Kee, 2011). It is well demonstrated that by 2020 , half of the US population will live at least with one chronic disease and researches conducted in other countries such as England, Canada, Australia, China and Iran suggest that chronic diseases are increasing among the population (Jordan \& Osborne, 2007), and some of these chronic diseases cause muscle weakness and atrophy. One of the chronic diseases that are prevalent in Iran and the world is multiple sclerosis which is a progressive neurological disorder, often disabling and unexplained, which affects the central nervous system, the brain and spinal cord) Mohr \& Pelletier, 2006). This disease is the leading cause of neurological disability in young and middle-aged people and has influenced almost one million young adults worldwide (William, Rigby, Airey, Robinson \& Ford, 1995). The prevalence of MS is higher in women than men and the most common ages are between the 20 to 40 years old. Association between these two, that means the involvement of youth and the disabling effect in childbearing age, show the importance of this disease and the need for more research in this field. In this disease, the myelin sheath of nerve cells in the brain and spinal cord are damaged. This damage has interrupted in parts of the nervous system's ability which are responsible for communication, and causes

Correspondence:

Montenegro Hamid Arazi

Gport University of Guilan, Department of Exercise Physiology, Faculty of Sport Sciences, P.O. Box: 41635-1438, Rasht, Iran E-Mail: hamidarazi@yahoo.com 
physical symptoms and health problems (Freeman, Fox, Gear \& Hough, 2012).

The main problem in patients with MS is the weakness and limitation of movement. Factors such as restricted movement, impatience, depression and fear of falling lead to limited participation of MS patients in physical activity. Physical inactivity causes muscle weakness and failure, constipation and bedsores (Dalgas, Stenager \& Ingemann-Hansen, 2008). Performing special physical activities has important role in the activities of daily living, mental health, social relationships and self-reliance of MS patients (Dalgas et al., 2008). Asano (2010) examined the effects of exercise and physical activity among persons with MS, the results showed that physical activity used, eventually led to higher levels of self-confidence, ability to promote active living, strength, endurance and flexibility, besides no injuries, accidents, or extreme fatigue due to exercise were reported (Asano, 2010).

Considering movement disorders and also cost of medications, it seems that exercise is one of the effective and inexpensive ways that has no unwanted side effects in treating the MS patients, so the relationship between physical activity and MS patients deserves further investigation. Hand grip strength is the force applied by the hand to pull on or suspend from objects (Sartorio, Lafortuna, Pogliaghi \& Trecate, 2002). Hand grip strength is linked to the upper extremity strength, general power and some anthropometric characteristics, so, often accepted as an objective assessment of upper limb function in clinical treatment (Balogun, Akomolafe \& Amusa, 1991). In previous studies, strong significant correlation has been reported between the hand grip strength and muscle mass (Steff et al., 2015). Since the hand grip strength is the representative of different physiological systems, its strengthening could be considered a practical strategy for improving public health and makes it less likely to have multiple chronic diseases and as a result, reduce premature deaths (Cheung, Nguyen, Au, Tan \& Kung, 2013). The 2D:4D ratio as an indicator of the balance between prenatal testosterone and estrogen is lower in male than females and this ratio remains stable throughout the life. This stability is due to prenatal testosterone exposure in humans and mammals and comprehensively is being used as a predictive indicator for assessing the impact of androgen hormones before birth in humans, such as physical strength (Kilduff et al., 2013). The role of testosterone as the most important anabolic hormone is very important, it stimulates the protein synthesis and its role in the growth and maintenance of muscle tissue is very important (Kreamer et al., 2001) Male sex hormones can have nerve protective effect. There are abundant evidence indicating the role of testosterone in immune response and effect on nerve cell regeneration following injury (Shuster, 2008).

Since the 2D:4D ratio is indicative of anabolic hormones, it may predict the possibility of some chronic diseases, in which muscle strength is reduced. Moy et al (2011) concluded that height, weight and race are significant predictors of elderly males and females (Moy et al., 2011). Hand grip strength is influenced by various factors such as gender, age and socioeconomic status (Leyk et al., 2007). Manning et al (2000) reported that the 2D:4D ratio is significantly different among different populations and genders (Manning et al., 2000). Since to the best of our knowledge there is only one study about the relationship between the 2D:4D ratio with MS disease in available resources (Kordi et al., 2014) and besides, factors such as increase in the prevalence of MS, increased mortality, very high medical costs, various problems, especially disruption of normal daily activities (which limits the daily activities in $69 / 4$ percent of patients), it seems essential to do further studies about the possible factors affecting the outbreak of the disease for coping with side effects and also provide practical suggestions in order to prevent its occurrence. Also, given that physical activity of hand grip strength and 2D:4D ratio measurements are simple and according to the literature may be helpful in identifying people susceptible to MS disease, this research was conducted to study the relationship between hand grip strength and 2D:4D ratio with the incidence of multiple sclerosis, so maybe with the supposed achievements, reducing health care costs, diagnosis of the disease in its early stages, reducing the enormous costs of disease in the future stages, faster access of patients to medicines, more practical methods of treatment, reduction of intensive care will be provided.

\section{Methods}

\section{Subjects and Experimental Approach}

This cross-sectional study was conducted on 100 young patients with MS, among the patients admitted to Shariati, Imam Hussein and Prophet Muhammad (PBUH) hospitals in Tehran in time intervals of December 2015 to June 2016. The subjects were informed about the possible risks and benefits of the study and gave their informed consent to participate in this study. Subjects' height, body weight and body mass index were determined. Isometric Dynamometer was used for the determination of grip strength. This study was approved in advance by the Institutional Ethics Review Committee from the University.

\section{Procedures}

\section{Anthropometric Measurements}

The body height of the patients was measured by a tape measure with an accuracy of $\pm 1 \mathrm{~cm}$ and electronic scales (SECA, Germany) accurate to within $0.1 \mathrm{~kg}$ were used to measure body mass and and body mass index (BMI), using bodyweight in kilograms divided by height in meters squared.

\section{Physical activity level}

Physical activity level was evaluated using a physical activity questionnaire (PAQ) which included 23 questions divided into three parts (work, exercise and leisure) which measure the individual's physical activities at work, sports and leisure time. There were eight questions about one's physical activity at job, eight questions about exercise that he/she is doing (its duration and intensity) and seven questions about the individual's activity during leisure time. Activities such as billiards, bowling, golf was considered as low intensity, badminton, cycling, swimming, ping pong as moderate intensity and boxing, basketball, football, rowing was considered as high intensity. Ones who had regular physical activity in recent years and were active during work and leisure, their index of physical activity by the questionnaire was about 8 to 10 and the physical activity index of inactive people, was about 4 to 5.5 . The expanded disability status scale (EDSS) score was determined by neurologist.

\section{Hand grip strength measurement}

Isometric Dynamometer was used for the determination 
of hand grip strength. Muscular strength was assessed using a digital strength dynamometer (SAEHAN corporation, Korea, model SH5000DP). The test was carried out while the subjects sat on a chair with a straight arm position. The test was performed three times within 30 seconds and the maximum strength was recorded.

\section{D:4D ratio measurement}

The ratio of right and left hand fingers assessment was conducted according to the method by (Manning, Scutt, Wilson \& Lewis-Jones, 1998). The length of the index finger (2D) and ring (4D) was considered from proximal fold to the tip of the fingers and was measured by digital caliper (digimatic caliper, Mitutoyo, China, model: 500-151-20) with an accuracy of $0 / 01 \mathrm{~mm}$. After measuring the length of the fingers, measurements were repeated again and the average of two measurements was recorded. The 2D:4D ratio is calculated by dividing the length of the index finger of a given hand by the length of the ring finger of the same hand.

Statistical analyses

Means and standard deviations are given as descriptive statistics. Following the determination of the normal distribution of data using Kolmogorov- Smirnov and after determining the feasibility of using parametric statistics, the Pearson correlation coefficient was used. The statistical significance was set at $\mathrm{p}<0.05$ presented as mean \pm standard error (SE) of the mean.

\section{Results}

Descriptive characteristics and isometric strength measurements of the patients in the study are presented in Table 1. As can be seen from Table 1, subjects were with a mean age of $(35.1 \pm 9.26$ years $)$ and BMI $\left(24.04 \pm 3.68 \mathrm{~kg} / \mathrm{m}^{2}\right)$ and EDSS in the range of 0 to 8.5 .

Table 1. Descriptive Characteristics of Participants

\begin{tabular}{lc}
\hline Variable & Mean \pm SD \\
\hline Body weight $(\mathrm{kg})$ & $63.04 \pm 10.25$ \\
Height $(\mathrm{m})$ & $1.61 \pm 0.05$ \\
Age (years) & $35.10 \pm 9.26$ \\
Body mass index $(\mathrm{kg} / \mathrm{m} 2)$ & $24.04 \pm 3.68$ \\
2D:4D ratio of right hand & $1.011 \pm 0.031$ \\
2D:4D ratio of left hand & $1.010 \pm 0.030$ \\
HGS of right hand & $21.40 \pm 6.44$ \\
HGS of left hand & $19.27 \pm 6.45$ \\
Level of disability (EDSS) & $2.54 \pm 2.11$ \\
Physical activity level & $6.80 \pm 1.11$ \\
\hline
\end{tabular}

SD: standard deviation; 2D:4D: second digit to fourth digit; HGS: hand grip strength; EDSS: expanded disability status scale

The results showed that there is a significant relationship $(r=-0.322$ and $p=0.001)$ between the physical activity level (A), hand grip strengh of right $(r=-0.226$ and $p=0.024)$ and left hand $(r=-0.202$ and $\mathrm{p}=0.044)$ with the disease (B and $\mathrm{C})$. However there is not a significant relationship between the
$2 \mathrm{D}: 4 \mathrm{D}$ ratio of right $(\mathrm{r}=-0.018$ and $\mathrm{p}=0.856)$ and left hand $(\mathrm{r}=$ -0.037 and $p=0.714$ ) with the disease (D and $E$ ) but the hand grip strengh of right $(\mathrm{r}=-0.252$ and $\mathrm{p}=0.011)$ and left hand $(r=-0.231$ and $p=0.021)$ have significant relationship with physical activity level ( $F$ and $G)$.

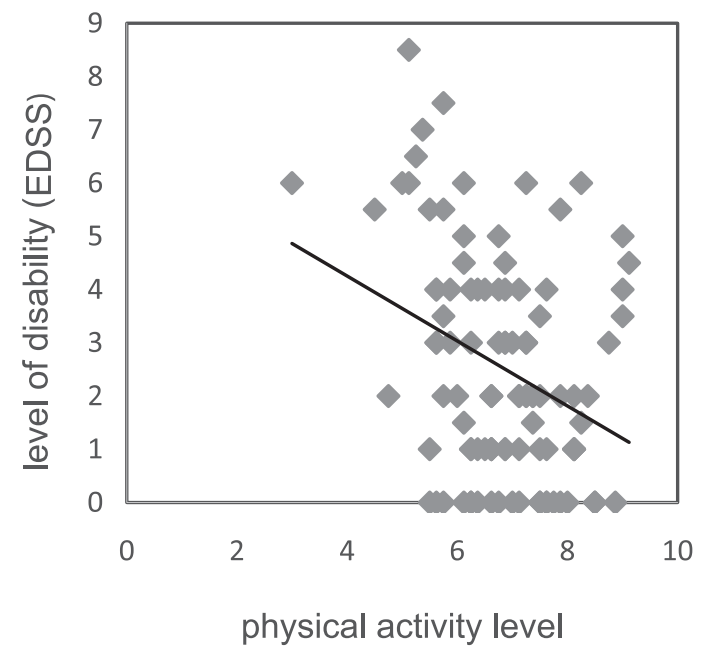

(A) 


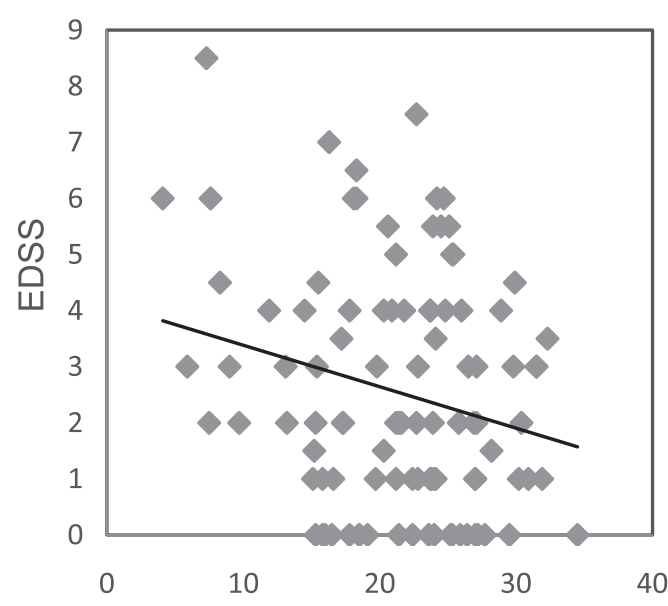

Hand grip strength of right hand

(B)

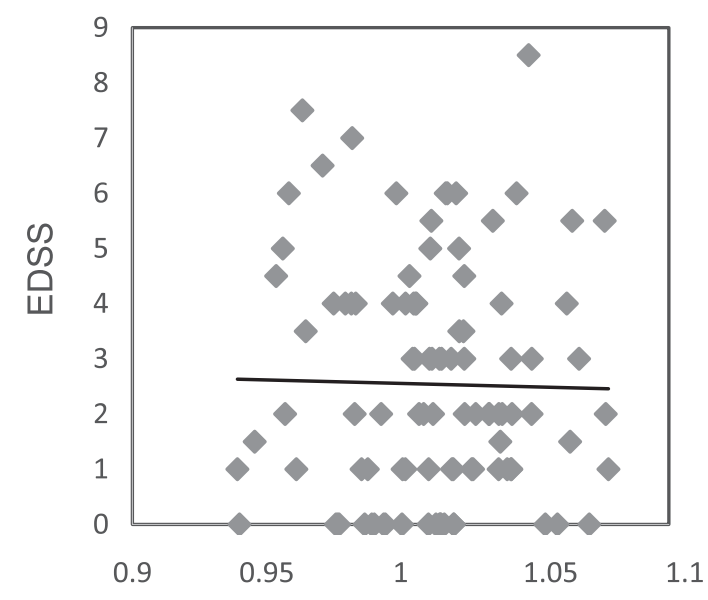

$2 D$ to $4 D$ ratio of right hand

(D)

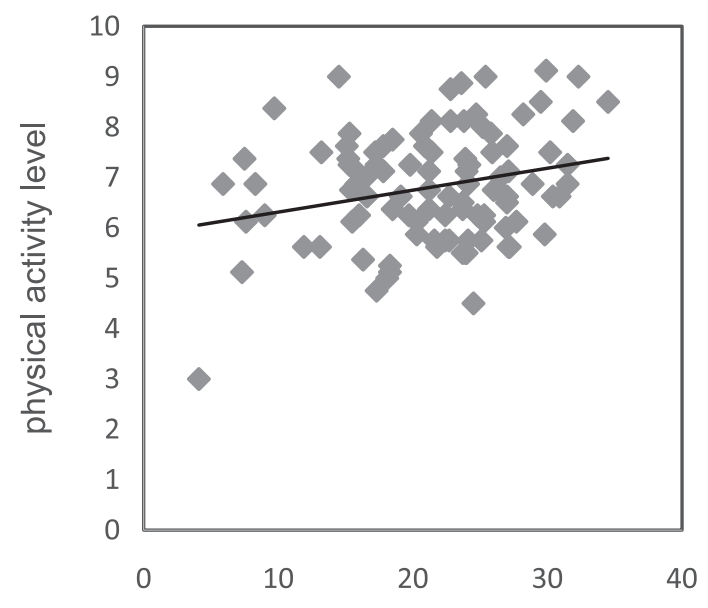

hand grip strength of right hand $(\mathrm{kg})$

(F)

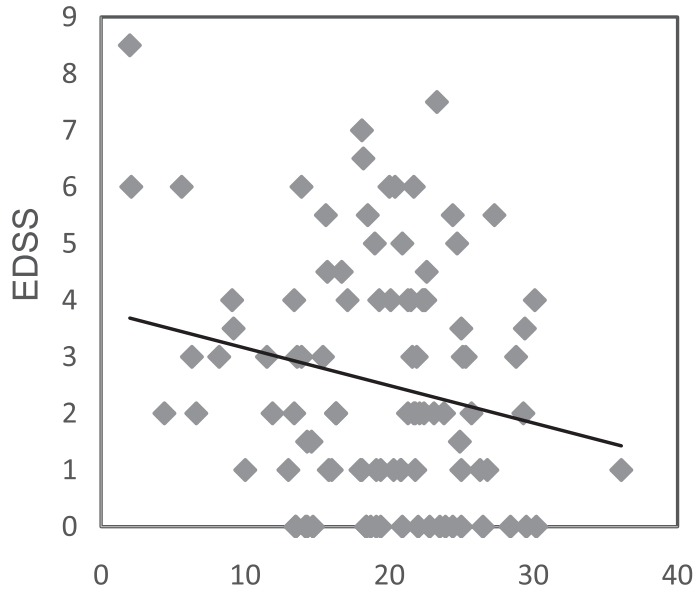

Hand grip strength of left hand

(C)

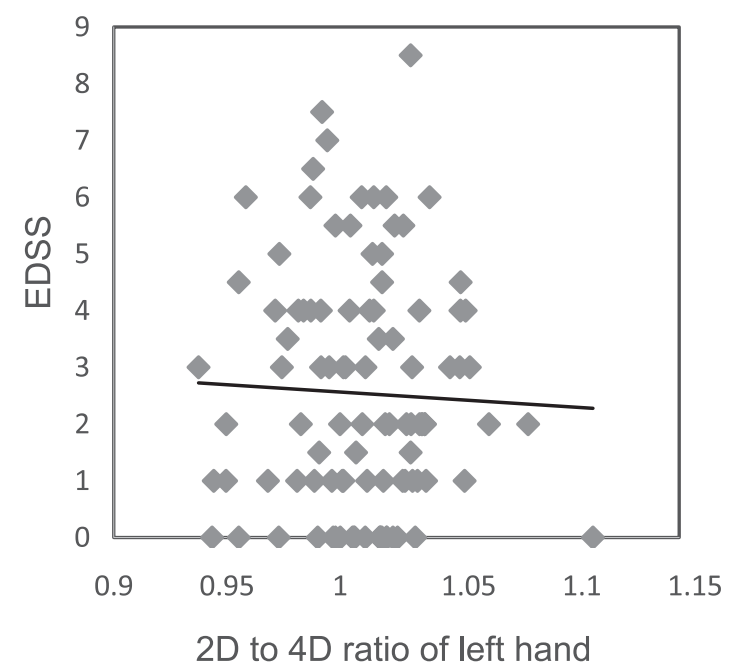

(E)

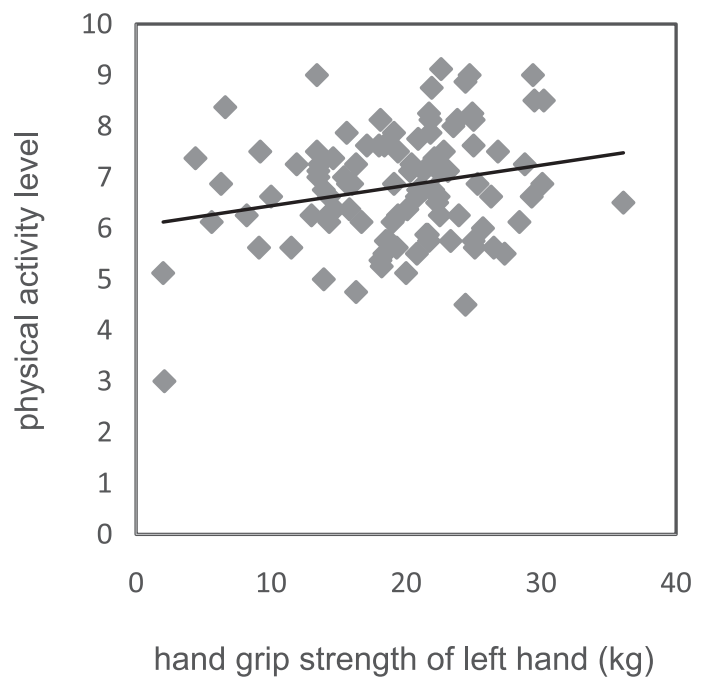

(G)

Figure 1. The relationship between the physical activity level and EDSS (A). The relationship between grip strength of right hand and EDSS (B). The relationship between grip strength of left hand and EDSS (C). The relationship between 2D:4D ratio of right hand and EDSS (D). The relationship between 2D:4D ratio of left hand and EDSS (E). The relationship between grip strength of right hand and physical activity level (F). The relationship between grip strength of left hand and physical activity level (G). 


\section{Discussion}

The results of the study showed that, patients who are physically active have lower level of disability (EDSS). Many studies have shown that physical activity improves the physical abilities of MS patients. Many studies have confirmed that exercise in mild-to-moderate MS patients has similar physical and psychological benefits as healthy control group. Recent studies have stated that exercise can cause numerous health consequences such as improving cardiorespiratory and muscular function and reduce depression and fatigue which are promoting quality of life and healthy living in patients. Exercise adjusts the immune responses by producing cytokines, which are involved in the regulation of immune and inflammatory responses. Several studies have been conducted on the effects of short-term exercise on cytokine production in healthy population (Maghsoudi, Nazarali, Khosravi \& Ravasi, 2011). While, the effect of exercise on the immune system of MS patients remains unknown and little research has been conducted in this area.

The results about the effects of exercise on disability (EDSS) of MS patients are different. Golzari, Shabkhiz, Soudi, Kordi \& Hashemi, 2010; Castellano, Patel \& White, 2008) showed that physical activity improves patients' clinical symptoms while the result of (Bjarnadottir, Konradsdottir, Reynisdottir \& Olafsson, 2007) was opposed. Golzari et al (2010) examined the effect of 24 sessions combined exercise training over eight weeks on 20 MS patients between the ages of 20 and 50 with disability status scale (EDSS) of 0-4. After eight weeks of exercise training, EDSS significantly decreased in the combined exercise group. Castellano et al (2008) studied the effects of $30 \mathrm{~min}$ aerobic exercise at $60 \%$ VO2peak, three days a week for eight weeks on MS patients' EDSS level. The results of their research showed that MS patients' EDSS level reduced 24\% (from 3.4 to 2.6) after eight weeks of aerobic exercise. While Bejrandotir et al (2007) examined the effect of five weeks of combined aerobic and strength training, three times a week on $16 \mathrm{MS}$ patients and finally, didn't find significant changes in MS patients' EDSS level. Probably the reason for conflicts of these studies is differences in the duration and intensity of exercise training programs. Based on the results, patients with high hand grip strength had lower EDSS level. There is much scientific evidence on the effectiveness of internal factors such as body composition, physical size, the superior hand and upper extremity anthropometric characteristics and external factors such as physical activity and health status on hand grip strength (Tiainen et al., 2004). Herein, the prognostic value of grip strength refers to its ability to forecast future outcomes. Grip strength is well established historically in this regard. Numerous studies published over the past several years have reinforced grip strength as a robust predictor of mortality, hospital length of stay, and physical functioning (Bohannon 2015) (Fig. 2).

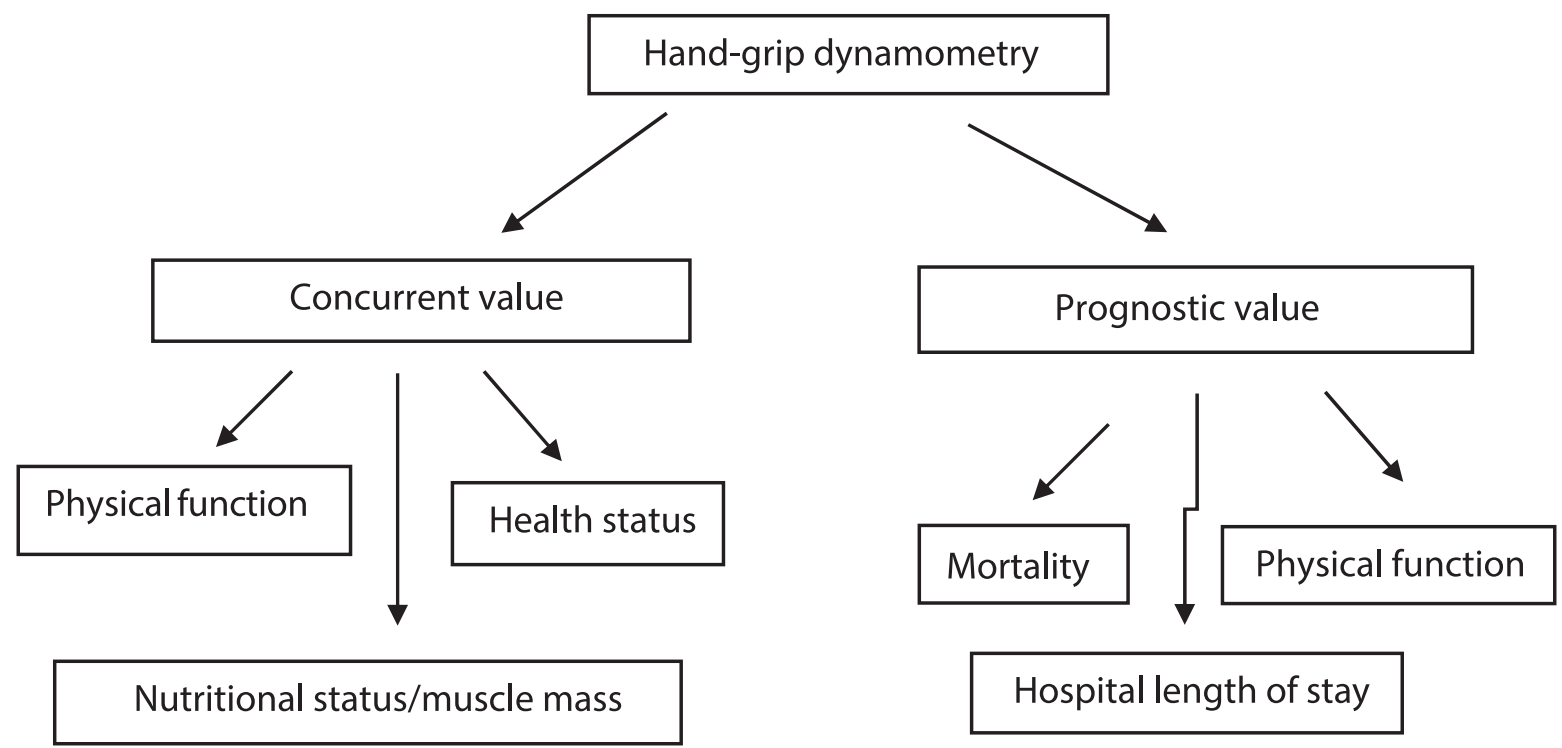

Figure 2. hand grip strengh as an indicator of contemporary and future situation

Since most of daily living activities are the main indicators of independence and are associated with manual dexterity, the patient can increase hand grip strengh by appropriate exercise and muscle strengthening movements to improve the quality of life and reduce EDSS level. In this study it was found that the 2D: 4D ratio was not related to the intensity (level) of disease in females suffering MS which is consistent with the research by Bove et al (2015) which was conducted on men suffering MS and found that the 2D: 4D ratio was higher in MS patients than the without MS group and this ratio is not associated with age and testosterone level in adults or clinical characteristics of MS. Disorders of the immune system which is separated into two branches of humoral and Cellular immunity can result in autoimmune diseases. MS is considered to be an immune-mediated disease in which the body's cell-mediated immune process is affected and are influenced by T helper type 1 (Th1) producing TNF $\beta$ (Tumor necrosis factor $\beta$ ), gamma interferon and interleukin-2 (IL2). On the other hand, T helper type 2 (Th2) cells activate IL-4, IL-5, IL6 and IL-10 that activate humoral immunity. When worsened MS symptoms clear up, the role of cellular immunity by cytokine secretion throughTh1 becomes more prominent, whereas in times of reduced symptoms, this response changes towards cytokine secretion through Th2 (Correale 1995). Since the sex steroids change the balance between Th1 and Th2 cells, MS disease activity can also be changed by altering the level of 
sex steroid hormones. This status is similar during pregnancy and after menopause in patients (Faas et al., 2000; Formby, 1995). Some studies have also proven the shift toward humoral immunity and Th2 during pregnancy due to high levels of estrogen (Formby, 1995). So that using the low power estrogen estriol therapy, which is greatly produced during pregnancy, is effective in reducing the size and number of lesions seen in MRI images of patients (Sicotte et al., 2002). Suggestive evidence indicates the relationship between MS disease and female sex hormones. The main concept of this study is that androgens before birth, may affect the risk of developing MS in women.

Some gender differences in this disease may be caused by sex hormones, including estrogen, progesterone and testosterone. Today the treatment with these hormones is highly regarded because they play an important role in modulating the immune system, neuroprotection and myelin repair functions. A sex steroid such as testosterone affects brain cells function by protecting central nervous system cells through mitochondrial stabilization, and protects cells against cell death. In animal models estrogen, testosterone and progesterone can affect the disease by modulating the immune system and inflammatory processes. Most body's immune system organizations have sex hormone receptor. It has been shown that in animals, testosterone against multiple sclerosis like diseases and other autoimmune diseases have protective role. The interaction between the immune system, endocrine, neurological and genetic factors is a possible reason for the effect of sexual hormones on susceptibility to multiple sclerosis.

The protective role of testosterone in autoimmune processes have been shown in animal models which in the presence of high levels of this hormone, killer lymphocyte cells exposed to autoantigens makes higher amounts of IL-5 and IL-10 and TNF production quantities are reduced. The free form of this hormone cross the blood-brain barrier and thus directly affect the nerve cells and preserves the culture medium of these cells from the toxic effects of glutamate. In general, empirical and laboratory evidence and magnetic imaging results show the relationship between sex steroid hormones (including estrogen, testosterone, progesterone and dehydroepiandrosterone) and multiple sclerosis (Foroughi pour, Norbakhsh, Hosseinpour Najafabadi \& Memar, 2012). Mechanistically, given reported anti-inflammatory and neuroprotective effects in animal models, a higher androgen to estrogen ratio might have an organizing role in modulating downstream inflammatory responses. The relationship between the 2D: 4D ratio and different phenotypes in adults has been investigated. The strongest phenotypes included the lower 2D: 4D which was raised in connection with athletic abilities and autism spectrum disorder. In neurological diseases, men with the higher 2D: 4D ratios have a higher risk for Alzheimer's disease. While the lower 2D: 4D ratio in females was associated with the risk of Alzheimer. In contrast, the lower 2D: 4D ratio is associated with the higher risk of amyotrophic lateral sclerosis. Recently it has been shown that a higher 2D: 4D ratio is associated with higher levels of inflammatory marker (interleukin-6) in girls aged 9 to 13 years, while there is not published data for its relationship with autoimmune diseases. ${ }^{29}$ In the present study as a second evaluation on the relationship between the 2D: $4 \mathrm{D}$ ratio and MS, only females were studied. To ensure the results of the study, there is a need for further investigations about the relationship between 2D: $4 \mathrm{D}$ ratio and inflammatory markers, etc. in MS patients.

\section{Conclusion}

According to the results of this study, physical activity can improve the clinical symptoms of patients suffering multiple sclerosis and improve quality of life and tolerance facing the everyday problems. Therefore, effective exercise prescription should include recommendations on frequency, intensity, type, time, and progression of exercise that follows MS disease-specific guidelines and target improvements in hand grip strength to lower EDSS level of patients.

\section{Acknowledgements}

The authors gratefully acknowledge all the patients who took part in this study for their cooperation.

\section{Conflict of Interest}

The authors declare that there are no conflict of interest.

Received: 15 April 2018| Accepted: 16 June 2018| Published: 13 July 2018

\section{References}

Asano M. (2010). Promoting Exercise and Physical Activity among Persons with Multiple Sclerosis, Unpublished Doctoral Dissertation. Montreal, Q: McGill University.

Balogun JA, Akomolafe CT, Amusa LO. (1991). Grip strength: effects of testing posture and elbow position. Arch Phys Med Rehabil, 72(5), 280-3.

Bjarnadottir OH, Konradsdottir AD, Reynisdottir K, Olafsson E. (2007). Multiple sclerosis and brief moderate exercise. A randomised study. Mult Scler, 13(6), 776-82.

Bohannon RW. (2015). Muscle strength: clinical and prognostic value of hand-grip dynamometry. Curr Opin Clin Nutr Metab Care, 18(5), 465-70.

Bove R, Malik MT, Diaz-Cruz C, Chua A, Saraceno TJ, Bargiela D, Greeke E, Glanz BI, Healy BC, Chitnis T. (2015). The 2D: 4D ratio, a proxy for prenatal androgen levels, differs in men with and without MS. Neurology, 85(14), 1209-13.

Castellano V, Patel DI, White LJ. (2008). Cytokine responses to acute and chronic exercise in multiple sclerosis. J Appl Physiol, 104(6), 1697-702.

Cheung CL, Nguyen US, Au E, Tan KC, Kung AW. (2013). Association of handgrip strength with chronic diseases and multimorbidity. Age, 35(3), 92941.

Correale J, Gilmore W, McMillan M, Li S, McCarthy K, Le T, Weiner LP. (1995). Patterns of cytokine secretion by autoreactive proteolipid proteinspecific $T$ cell clones during the course of multiple sclerosis. J Immunol, 154(6), 2959-68.

Dalgas U, Stenager E, Ingemann-Hansen T. (2008). Multiple sclerosis and physical exercise: recommendations for the application of resistance-, endurance-and combined training. Mult scler, 14(1), 35-53.

Faas M, Bouman A, Moesa H, Heineman MJ, de LL, Schuiling G. (2000). The immune response during the luteal phase of the ovarian cycle: a Th2type response? Fertil Steril, 74(5), 1008-13.

Formby B. (1995). Immunologic response in pregnancy. Its role in endocrine disorders of pregnancy and influence on the course of maternal autoimmune diseases. Endocrinol Metab Clin North Am, 24(1), 187-205.

Foroughi Pour A, Norbakhsh V, Hosseinpour Najafabadi S, Memar R. (2012). Evaluating sex hormone levels in reproductive age women with multiple sclerosis and their relationship with disease severity. $J$ Res Med Sci, 17(9), 882-85.

Freeman J, Fox E, Gear M, Hough A. (2012). Pilates based core stability training in ambulant individuals with multiple sclerosis: protocol for a multi-centre randomised controlled trial. BMC Neurol, 12:19.

Golzari Z, Shabkhiz F, Soudi S, Kordi MR, Hashemi SM. (2010). Combined exercise training reduces IFN- $\gamma$ and IL-17 levels in the plasma and the supernatant of peripheral blood mononuclear cells in women with multiple sclerosis. Int Immunopharmacol, 10(11), 1415-19.

Jordan JE, Osborne RH. (2007). Chronic disease self-management education programs: challenges ahead. Med J Aust, 186(2), 84-7.

Kilduff L, Cook CJ, Bennett M, Crewther B, Bracken RM, Manning J. (2013). Right-left digit ratio (2D: 4D) predicts free testosterone levels associated with a physical challenge. J Sports Sci, 31(6), 677-83.

Kordi MR, Anooshe L, Khodadade S, Maghsodi N, Sanglachi B, Hemmatinafar B. (2014). Comparing the Effect of Three Methods of Combined Training on Serum Levels of Ghrelin, Pro and Anti-inflammatory Cytokines in Multiple Sclerosis (MS) Patients. ZUMS Journal, 22(91), 39-51.

Kraemer WJ, Loebel CC, Volek JS, Ratamess NA, Newton RU, Wickham RB, Gotshalk LA, Duncan ND, Mazzetti SA, Gómez AL, Rubin MR, Nindl BC, 
Häkkinen K. (2001). The effect of heavy resistance exercise on the circadian rhythm of salivary testosterone in men. Eur J Appl Physiol, 84(1-2), 13-18.

Leyk D, Gorges W, Ridder D, Wunderlich M, Rüther T, Sievert A, Essfeld D. (2007). Hand-grip strength of young men, women and highly trained female athletes. Eur J Appl Physiol, 99(4), 415-21.

Maghsoudi N, Nazarali P, Khosravi N, Ravasi A. (2011). Effect of selected exercise (aerobic and resistance) to some of immune system factors in male patients with MS. Electronic Physician, 3(3), 346.

Manning JT, Barley L, Walton J, Lewis-Jones DI, Trivers RL, Singh D, Thornhil R, Rohde P, Bereczkei T, Henzi P, Soler M, Szwed A. (2000). The 2nd: 4th digit ratio, sexual dimorphism, population differences, and reproduc tive success: evidence for sexually antagonistic genes? Evol Hum Behav 21(3), 163-83

Manning JT, Scutt D, Wilson J, Lewis-Jones DI. (1998). The ratio of 2nd to 4th digit length: a predictor of sperm numbers and concentrations of testosterone, luteinizing hormone and oestrogen. Hum Reprod, 13(11), 3000-4.

Mohr DC, Pelletier D.) 2006. ( A temporal framework for understanding the effects of stressful life events on inflammation in patients with multiple sclerosis. Brain Behav Immun, 20(1), 27-36.
Moy F, Chang E, Kee K. (2011). Predictors of handgrip strength among the free living elderly in rural Pahang, Malaysia. Iranian J pub health, 40(4), 44-53.

Sartorio A, Lafortuna CL, Pogliaghi S, Trecate L. (2002). The impact of gender, body dimension and body composition on hand-grip strength in healthy children. J Endocrinol Invest, 25(5), 431-5.

Shuster, EA. (2008). Hormonal influences in multiple sclerosis. Curr Top Microbiol Immunol, 318: 267-311.

Sicotte NL, Liva SM, Klutch R, Pfeiffer P, Bouvier S, Odesa S, Wu TC, Voskuhl RR. (2002). Treatment of multiple sclerosis with the pregnancy hormone estriol. Ann Neurol, 52(4), 421-8.

Steff M, Bohannon R, Houdova V, Musalek M, Prajerova K, Cesak P, Petr M, Kohlikova E, Holmerova I. (2015). Relationship between clinical measures of sarcopenia in a sample of community-dwelling women. Isokin Exerc Sci, 23(1), 41-44.

Tiainen K, Sipila S, Alen M, Heikkinen E, Kaprio J, Koskenvuo M, Tolvanen A Pajala S, Rantanen T. (2004). Heritability of maximal isometric muscle strength in older female twins. J Appl Physiol, 96, 173-180.

William R, Rigby AS, Airey M, Robinson M, Ford H. (1995). Multiple sclerosis: its epidemiological, genetic, and health care impact. J Epidemiol Community Health, 49, 563-9. 